\title{
Efficient Computation of Generalized Bessel Function Distributions
}

\author{
Ilir F. Progri \\ Giftet Inc., 5 Euclid Ave. \#3, Worcester, MA 01610, USA \\ ORCID: 0000-0001-5197-1278
}

Correspondence should be addressed to Ilir Progri; iprogri@giftet.com

Received March 23, 2017; Revised March 24, 31, 2017-June 7, 2018, Accepted July 16, 2018; Published November 1, 2018.

Scientific Editor-in-Chief/Editor: Ilir F. Progri

Copyright (C) 2018 Giftet Inc. All rights reserved. This work may not be translated or copied in whole or in part without written permission to the publisher (Giftet Inc., 5 Euclid Ave. \#3, Worcester, MA 01610, USA), except for brief excerpts in connection with reviews or scholarly analysis. Use in connection with any form of information storage and retrieval, electronic adaptation, computer software or by similar or dissimilar methodology now known or hereafter developed is forbidden. The use of the publication of trade names, trademarks, service marks, or similar terms, even if they are not identified as such, is not to be taken as an expression of opinion as to whether or not they are subject to proprietary rights.

This paper discusses an efficient computation of two generalized Bessel function distributions one of which is original and never presented before in the literature. For each generalized Bessel distribution model the closed form expression of the cumulative distribution function (cdf) is given by means of Legendre continued fraction and Taylor series expansion of the modified Bessel functions of the first kind, for both integer and real values of a parameter, and of the second kind, for real values of the same parameter, which leads to incomplete gamma functions, hypergeometric series, the Kampé de Fériet function (or double hypergeometric series). Efficient computations of the algorithms (which are implemented in MATLAB functions) are also presented in the paper. Numerical results are derived for each case to validate the theoretical models presented in the paper.

Index Terms-Bessel functions, modified Bessel functions, cumulative distribution function, Kampé de Fériet function, series expansion, closed form expression, signal analysis, generalized functions, time-domain analysis, incomplete gamma functions, hypergeometric series.

\section{Introduction}

The first original closed form expression of the generalized Bessel function cumulative distribution functions (cdf) of the first and second kinds were first produced by Progri $2016[1]^{\mathrm{i}}$.

The issue, however, is that the efficient computation of these cdfs require the computation of the Kampé de Fériet function (or double hypergeometric series [1]-[13]) in MATLAB. The main issue is the following: Is there an algorithm that we can apply to successfully produce an efficient MATLAB function of the Kampé de Fériet function (or double hypergeometric series [1]-[13])? Is this algorithm also computationally stable?

Before we go further into the discussion of the material of this journal paper it is important to mention a bit more 
background research work.

The efficient computation of generalized Bessel function distributions cdfs is intrinsically linked to the efficient computation of the incomplete gamma functions [12], [21]-[34] which is intrinsically linked to the efficient computation of Taylor series ${ }^{\text {ii }}$ and the Legendre continued fraction [16], [19].

As reported by Bateman 1953-1955 [13] (vol. II pg. 134), the investigation of the incomplete gamma function first begun with Legendre (1811, [17] pp. 339-343 and later works).

It is not clear who performed the first series expansion of the incomplete gamma function by means of the Taylor series [14][15]. It could be that Legendre [16]-[18] either did it or compiled the work that was performed before him. What is clear is that the Legendre continued fraction [16]-[18] is nearly a hundred years (or a century) later than the Taylor series expansion [14]-[15].

The efficient computation of the incomplete gamma functions has begun as early as Legendre [16]-[18], to Pearson 1922 [20], to Bateman 1953-1955 [13] (vol. pg. 133-1952) in form of tables, formulas, identities, etc. There is a reason why this form of publication is still very important today with the work of Gradshteyn, Ryzhik Table of Integrals, Series, and Products 2007 [36]. Nevertheless, no matter how important the table or tables are when the exact computation of a function is required, it is important to develop the full computational algorithm.

From 1811 to $1972-1979$ there was a lot of published material on the properties of the incomplete gamma function [13]-[20]. The references on this paper present a small fraction of all the contributors on this subject.

What is clear is that with various publications of Gautschi listed as references in Gautschi 1979 [21] we see various development stages of a fully computational algorithm, of the incomplete gamma functions. Gautschi 1979 [21] algorithms of the efficient computation of the incomplete gamma function consists on the primary function evaluation by means of the Taylor's series [14]-[15] and a combination of methods, including direct evaluation based partly on power series, recursive computation, and the classical continued fraction of Legendre [16]-[18]. Nevertheless, Gautschi 1979 [21] does on guaranty that his procedure is valid (or will strain) throughout the region $\mathcal{H}:\{(x, a): x \geq 0,-\infty<a<\infty\}$, for excessively large values of $a$ and $x$ particularly when $a \neq x \gg 1$ (cf. Section 5). In such cases Gautschi 1979 [21] proposed that it may be preferable to use asymptotic methods, e.g. the uniform asymptotic expansions of Temme 1977-1979 [22] (later publication of Temme 1985 [26], A.R DiDonato and A.H. Morris, Jr. 1986 [25] includes the computation of the incompete gamma function inverses from Temme's uniform asymptotic expansions and Temme 1996 [26] includes negative values, and the complete refined algorithm in Gil, Ruiz-Antolín, Segura, and Temme 2017 [33]).

Jones and Thron 1986 [23] expand the work of Gautschi 1979 [21] on the efficient computation of the incomplete gamma functions in the complex domain.

Winitzki 2003 [27] employs Gautschi 1979 [21] expansions to provide an efficient computation of the incomplete gamma function to arbitrary precision.

Blahak 2010 [28] also refers to the expansions employed by Gautschi 1979 [21] as an efficient approximation of the incomplete gamma function without referencing Gautschi 1979 [21].

Bailey and Borwein 2015 [30] provide a more sophisticated interpretation of the Gautschi 1979 [21] algorithms as an expansion of the Crandall's computation of the incomplete Gamma function by means of Laguerre polynomials.

Abergel and Moisan 2016 [31] refer to the Gautschi 1979 [21] algorithms as fast and accurate evaluation of the generalized incomplete gamma function.

Further analysis of the incomplete gamma functions are performed by Jameson 2016-2017 [32] to derive the two alternative expansions of Gautschi 1979 [21] without mentioning him as a reference.

Based on the work on Greengard and Rokhlin 2017-2018 [34] Numerical experiments show that in this regime, for all $x>0$, $a<10,000$ evaluation of $\gamma(a, x)$ using Gautschi 1979 [21] is faster than evaluation by asymptotic expansion (Temme 19771979 [22], Temme 1985 [26], A.R DiDonato and A.H. Morris, Jr. 1986 [25], Temme 1996 [26] Gil, Ruiz-Antolín, Segura, and Temme 2017 [33]).

It is without any doubt that Gautschi 1979 [21] algorithms (based on Legendre [16]-[18] and Taylor series [14]-[15]) are really the centerpiece of the efficient computation of generalized Bessel function distributions performed by Progri 2016 [1] and in the current publication.

This paper presents an original work that falls into the category of Marvels in analytical derivations and computational mathematics series never published anywhere else.

In 2016, the computation of cdfs of the Generalized Bessel 
Function Distributions was performed via the linear numerical approximation. As seen in this paper this method is grossly inefficient and marginally accurate. However, in 2016, it served as the first approximation and as the baseline of the efficient computation methods presented in this paper.

In this paper we are going to compare and contrast four cdf expressions of the Generalized Bessel Function Distributions.

The first expression is cdf that is obtained as a result of the numerical integration of the pdf of the Generalized Bessel function distributions. On the positive side this expression is very accurate. On the negative side it does not exist in closed form; i.e., it only exists in the integral form.

The second expression is the cdf that is produced from linear numerical approximation (or summation) of the pervious expression. This expression is grossly inefficient and marginally accurate. This expression was initially used as a verification method of the pdfs of the generalized Bessel function distributions in Progri [1].

The third cdf expression is obtained from computation of the closed form expression of the generalized Bessel function distributions by means of the Kampé de Fériet function (or double hypergeometric series from Gautschi 1979 [21] expansion of the incomplete gamma function via Legendre continued fraction [16]). Since the computation of the Kampé de Fériet function (or double hypergeometric series) is an essential part of the efficient computation Generalized Bessel Function Distributions; hence, we go much more in depth on the computation of the Kampé de Fériet function.

The forth cdf expression is obtained from the computation of any of the equations of the cdf in Progri [1]. This serves as the test to enable the end-to-end verification of the expressions produced in Progri [1]

The first attempt for producing a MATLAB function of the computation of the Kampé de Fériet function is given by Agarwal, Chand 2012 [11]. We consider this as the baseline option for the computation of the Kampé de Fériet function. There are two main issues with this baseline option for the computation of the Kampé de Fériet function as presented in Agarwal, Chand 2012 [11]. The first issue is that this function is numerically unstable; the second issue is that it does not converge as the number of terms increases typically beyond one hundred. In Appendix A, Dr. Progri, for the first time, presents a dramatically improved formulation of the Kampé de Fériet function that reduces the computation of the Kampé de Fériet function from a double summation to a single summation. This formulation is necessary because it partially takes care of the numerical stability or the convergence problem. It does not completely eliminate the numerical instability of the issues related to the convergence problem because it still employs some formulation of the option one. Furthermore, Dr. Progri for the first time provides a very efficient and computationally stable formulation of the of the Kampé de Fériet function that completely eliminates the numerical instability of the issues related to the convergence problem as illustrated further in Appendix A. This is known as option three in the computation of the Kampé de Fériet function. At this point the computation of the Kampé de Fériet function is numerically very stable, computationally very efficient, and numerically very accurate; i.e., the Kampé de Fériet function numerical evaluation in MATLAB is no longer impacted by the numerical stability, efficiency, and accuracy. I hope that the Kampé de Fériet function can be implemented in MATLAB in like manner as the generalized hypergeometric series function hypergeom $(\mathrm{a}, \mathrm{b}, \mathrm{z})$ [40].

In the numerical results section we present both the pdfs and cdfs of the generalized Bessel functions of the first and second kinds for values of $x$ from $-\infty$ to $\infty$. The methodology is consistent with the works presented by Arnaut 2003 [40] even though the work of the latter is only valid for positive xs and only for a special class of Bessel function distributions.

Since, the generalized Bessel functions cdfs require the computation of the Kampé de Fériet function [8]-[10] and the latter is not yet available in MATLAB 2016a [40], Giftet Inc. is also taking on the task to compute the Kampé de Fériet function [8]-[10] as part of the Indoor Geolocation Systems MATLAB Library and publish the results of this work in Dr. Progri pioneer publication [35].

This paper is organized as follows: in Sect. 2 efficient computation of the generalized Bessel function distribution CDF of the first kind is discussed. The efficient computation of the generalized Bessel function distribution CDF of the second kind is presented in Sect. 3. Section 4 contains numerical, theoretical results; Conclusion is provided in Sect. 5 along with a list of references. In Appendix A: A super-efficient implementation of the Kampé de Fériet function or double hypergeometric series is presented. 


\section{Efficient Computation of the Generalized Bessel Function Distribution CDF of the First Kind}

There are two fundamental definitions (or series expansions) of the incomplete gamma function (see Bateman 1953-1955 [13] (vol.pg. 133 (3); see Gradshteyn, Ryzhik [36] pg. 899 ex. 8.351 2.)

$$
\gamma(\alpha, x)=\frac{x^{\alpha} e^{-x}}{\alpha} \Phi(1,1+\alpha ; x)^{\mathrm{iii}}=\frac{x^{\alpha}}{\alpha} \Phi(\alpha, 1+\alpha ;-x)
$$

Where $\Phi(\alpha, \gamma ; x)$ is known as a confluent hypergeometric function (see Gradshteyn, Ryzhik [36] pg. 1023 ex. 9.210 1.)

$$
\Phi(\alpha, \gamma ; x)={ }_{1} F_{1}(\alpha ; \gamma ; x)=\sum_{n=0}^{\infty} \frac{(\alpha)_{n}}{(\gamma)_{n}} \frac{x^{n}}{n !}
$$

Substituting (2) into (1) produces

$$
\gamma(\alpha, x)=\frac{x^{\alpha} e^{-x}}{\alpha} \sum_{n=0}^{\infty} \frac{(1)_{n}}{(1+\alpha)_{n}} \frac{x^{n}}{n !}=\frac{x^{\alpha}}{\alpha} \sum_{n=0}^{\infty} \frac{(\alpha)_{n}}{(1+\alpha)_{n}} \frac{(-x)^{n}}{n !}(3)
$$

In Progri 2016 [1] we utilized the first series expansion of the incomplete gamma function.

It is important to investigate both definitions (or series expansion of the incomplete gamma function); i.e., provide the efficient computations for both series expansion of the incomplete gamma function that will ultimately result in the computation of the Kampé de Fériet function (or double hypergeometric series) [8]-[10].

The main purpose of this section is to show all the derivations that are required for producing (116) from (115) in Progri 2016 [1].

Equation (115) in (Progri 2016 [1]) is equivalent with $\alpha=$ $2 p+2 k$

$$
\gamma\left(2 p_{1}+2 k, d x\right)=\frac{(d x)^{2 p_{1}+2 k} e^{-d x} \sum_{n=0}^{\infty} \frac{(1)_{n}(d x)^{n}}{\left(2 p_{2}+2 k\right)_{n} n !}}{2 p_{1}+2 k}
$$

Next, we need to simplify the (rising) Pochhammer symbol $\left(2 p_{2}+2 k\right)_{n}[39]$ as follows

$$
\left(2 p_{2}+2 k\right)_{n}=\frac{\Gamma\left(2 p_{2}+2 k+n\right)}{\Gamma\left(2 p_{2}+2 k\right)}
$$

Next, based on the properties of the gamma function [37] we have the following

$$
\Gamma\left(2 p_{2}+2 k\right)=\frac{2^{2 p_{2}+2 k-1} \Gamma\left(p_{2}+k\right) \Gamma\left(p_{2}+k+\frac{1}{2}\right)}{\sqrt{\pi}}
$$

Which is equivalent with

$$
\Gamma\left(2 p_{2}+2 k\right)=\frac{2^{2 p_{14}}{ }^{k} \Gamma\left(p_{2}+k\right) \Gamma\left(p_{3}+k\right)}{\sqrt{\pi}}
$$

Similarly, we need to simplify $\Gamma\left(2 p_{2}+2 k+n\right)$ as follows, for $n$ even and odd. First, for $n=2 m$ even we have

$$
\Gamma\left(2 p_{2}+2 k+2 m\right)=\frac{4^{k+m} \Gamma\left(p_{2}+k+m\right) \Gamma\left(p_{3}+k+m\right)}{2^{-2 p_{1} \sqrt{\pi}}}
$$

and for $n=2 m+1$ odd we have

$$
\Gamma\left(2 p_{3}+2 k+2 m\right)=\frac{2^{2 p_{24}}{ }^{k+m} \Gamma\left(p_{3}+k+m\right) \Gamma\left(p_{4}+k+m\right)}{\sqrt{\pi}}
$$

Finally, substituting (8) and (7) into (5) for $n=2 m$ yields

$$
\left(2 p_{2}+2 k\right)_{n=2 m}=\frac{4^{m} \Gamma\left(p_{2}+k+m\right) \Gamma\left(p_{3}+k+m\right)}{\Gamma\left(p_{2}+k\right) \Gamma\left(p_{3}+k\right)}
$$

Which is equivalent with

$$
\left(2 p_{2}+2 k\right)_{n=2 m}=4^{m}\left(p_{2}+k\right)_{m}\left(p_{3}+k\right)_{m}
$$

Next, substituting (9) and (7) into (5) for $n=2 m+1$ yields

$$
\left(2 p_{2}+2 k\right)_{n=2 m+1}=\frac{2 \times 4^{m} \Gamma\left(p_{3}+k+m\right) \Gamma\left(p_{4}+k+m\right)}{2^{2 p_{1} \Gamma\left(p_{2}+k\right) \Gamma\left(p_{3}+k\right)}}
$$

Or equivalently

$$
\left(2 p_{2}+2 k\right)_{n=2 m+1}=\frac{\left(p_{3}+k\right)_{m}\left(p_{4}+k\right)_{m}\left(p_{2}+k\right)}{2^{-1} \times 4^{-m}}
$$

Equation (4) can be written as

where $p_{i}=p+\frac{i}{2} ; i=\{1,2,3,4\}$.

$$
\gamma\left(2 p_{1}+2 k, d x\right)=\frac{(d x)^{2 p_{1}+2 k} e^{-d x}}{2 p_{1}+2 k}\left[\sum_{\substack{n=0 \\ n=2 m}}^{\infty} \frac{(d x)^{2 m}}{\left(2 p_{2}+2 k\right)_{2 m}}+\sum_{\substack{n=2 m+1 \\ n=2 m+1}}^{\infty} \frac{(d x)^{2 m+1}}{\left(2 p_{2}+2 k\right)_{2 m+1}}\right]
$$

Next, substituting (11) into the first summation of (14) we obtain

$$
\sum_{\substack{n=0 \\ n=2 m}}^{\infty} \frac{(d x)^{2 m}}{\left(2 p_{2}+2 k\right)_{2 m}}=\sum_{\substack{n=0 \\ n=2 m}}^{\infty} \frac{(d x)^{2 m}}{4^{m}\left(p_{2}+k\right)_{m}\left(p_{3}+k\right)_{m}}
$$

Which is identical with

$$
\sum_{n=2 m}^{\infty} \frac{(d x)^{2 m}}{\left(2 p_{2}+2 k\right)_{2 m}}=\sum_{m=0}^{\infty} \frac{\left(\frac{d^{2} x^{2}}{2^{2}}\right)^{m}}{\left(p_{2}+k\right)_{m}\left(p_{3}+k\right)_{m}}
$$

Next, substituting (13) into the second summation of (14) yields

$$
\sum_{n=2 m+1}^{\infty} \frac{x^{2 m+1}}{\left(2 p_{2}+2 k\right)_{2 m+1}}=\frac{d x \sum_{n=2 m+1}^{\infty} \frac{\left(\frac{d^{2} x^{2}}{2^{2}}\right)^{m}}{2\left(p_{2}+k\right) m\left(p_{4}+k\right) m}}{2}
$$

Which is equivalent with 


$$
\begin{aligned}
\sum_{\substack{n=1 \\
n=2 m+1}}^{\infty} \frac{(d x)^{2 m+1}}{\left(2 p_{2}+2 k\right)_{2 m+1}} & =\frac{d x \sum_{m=0}^{\infty} \frac{\left.\left(\frac{d^{2} x^{2}}{2^{2}}\right)^{m}+k p_{m}+k\right)_{m}}{2\left(p_{2}+k\right)}}{\gamma} \\
& \gamma\left(2 p_{1}+2 k, d x\right)=\frac{(d x)^{2 p_{1}+2 k} e^{-d x}}{2 p_{1}+2 k}\left[\sum_{m=0}^{\infty} \frac{\left(\frac{d^{2} x^{2}}{2^{2}}\right)^{m}}{\left(p_{3}+k\right)_{m}\left(p_{2}+k\right)_{m}}+\frac{d x}{2\left(p_{2}+k\right)} \sum_{m=0}^{\infty} \frac{\left(\frac{d^{2} x^{2}}{2^{2}}\right)^{m}}{\left(p_{4}+k\right)_{m}\left(p_{3}+k\right)_{m}}\right]
\end{aligned}
$$

Substituting (19) into (88) of Progri 2016 [1] we obtain for $\quad \alpha=2 p+2 k$

$$
F_{\text {GBessel1 }}(x ; a, d, p)=\frac{\left(1-\frac{1}{d^{2}}\right)^{p_{1}} e^{-d x}(d x)^{2 p_{1}}}{\Gamma\left(2 p_{1}\right)} \sum_{k=0}^{\infty} \frac{x^{2 k}\left[\sum_{m=0}^{\infty} \frac{\left(\frac{d^{2} x^{2}}{2^{2}}\right)^{m}}{\left(p_{3}+k\right) m}+\frac{d x}{2\left(p_{2}+k\right) m} \sum_{m=0}^{\infty} \frac{\left(\frac{d^{2} x^{2}}{2^{2}}\right)^{m}}{\left(p_{4}+k\right)_{m}\left(p_{3}+k\right) m}\right]}{\left(2 p_{1}+2 k\right) k !\left(p_{2}\right)_{k} 2^{2 k}}
$$

Which is equivalent with the following

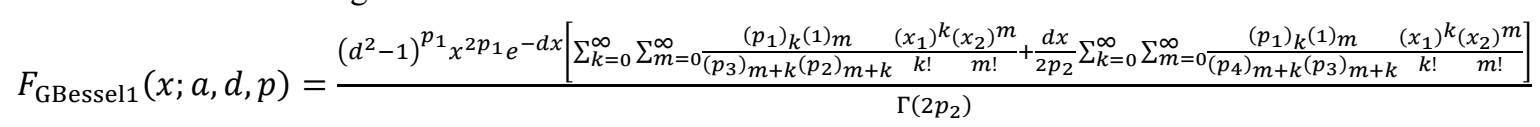

Where

$$
x_{1}=\frac{x^{2}}{4} ; x_{2}=\frac{x^{2} d^{2}}{4}
$$

The cdf of the Bessel function distribution of the first kind which can be written with the help of the Kampé de Fériet function (or double hypergeometric series) [8]-[10] as follows

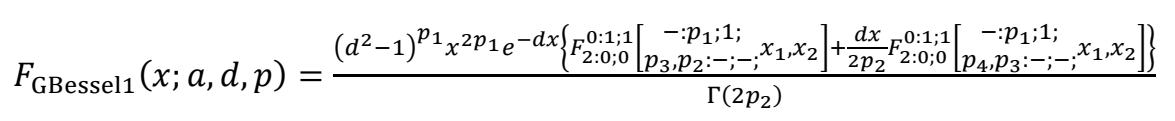

Which is equivalent with

$$
F_{\mathrm{GBessel} 1}(x ; a, d, p)=C_{11}(d, p) \mathcal{F}_{\mathrm{GBessel} 1}(x ; a, d, p)
$$

Where

$$
\mathcal{F}_{\mathrm{GBessel} 1}(x ; a, d, p)=\frac{x_{1}^{p_{1}} e^{-d x}}{\Gamma\left(p_{2}\right)}\left\{F_{2: 0 ; 0}^{0: 1 ; 1}\left[\begin{array}{c}
-: p_{1} ; 1 ; \\
p_{3}, p_{2}:-;-; x_{1}, x_{2}
\end{array}\right]+\frac{d x}{2 p_{2}} F_{2: 0 ; 0}^{0: 1 ; 1}\left[\begin{array}{c}
-: p_{1} ; 1 ; \\
p_{4}, p_{3}:--; x_{1}, x_{2}
\end{array}\right]\right\}
$$

$$
C_{11}(d, p)=\frac{\left(d^{2}-1\right)^{p_{1}} \Gamma\left(p_{2}\right)}{\Gamma\left(2 p_{2}\right)}
$$

Before the conclusion of this section we have shown that by comparing and contrasting (85) in Progri 2016 [1] with (24) the following is obtained

$$
\mathcal{F}_{\text {GBessel } 1}(x ; a, d, p)=d^{-2 p_{1}} \sum_{k=0}^{\infty} \frac{\gamma\left(2 p_{1}+2 k, d x\right)}{k ! \Gamma\left(p_{2}+k\right)(2 d)^{2 k}}
$$

which is equivalent with

$$
\mathcal{F}_{\text {GBessel } 1}(x ; a, d, p)=\frac{d^{-2 p_{1}}}{\Gamma\left(p_{2}\right)} \sum_{k=0}^{\infty} \frac{\gamma\left(2 p_{1}+2 k, d x\right)}{k !\left(p_{2}\right)_{k}(2 d)^{2 k}}
$$

This concludes the additional the derivations that are required for producing (116) from (115) in Progri 2016 [1].

\section{Efficient Computation of the Generalized Bessel Function Distribution CDF of the First Kind-Alternative Expansion}

The main purpose of this section is to show all the derivations that are required for producing the alternative expansion in Progri 2016 [1] see Bateman 1953-1955 [13] (vol. pg. 133 (3); see Gradshteyn, Ryzhik [36] pg. 899 ex. 8.3512.

$$
\gamma(\alpha, x)=\frac{x^{\alpha}}{\alpha} \sum_{n=0}^{\infty} \frac{(\alpha)_{n}}{(1+\alpha)_{n}} \frac{(-x)^{n}}{n !} \text { iv }
$$

Substituting $\alpha$ and $d x$ instead of $x$ into (29) yields,

$$
\gamma\left(2 p_{1}+2 k, d x\right)=\frac{(d x)^{2 p_{1}+2 k} \sum_{n=0}^{\infty} \frac{\left(2 p_{1}+2 k\right)_{n}(-d x)^{n}}{\left(2 p_{2}+2 k\right)_{n} n !}}{2\left(p_{1}+k\right)}
$$

Next, we need to simplify the (rising) Pochhammer symbol $\left(2 p_{1}+2 k\right)_{n}[39]$ as follows

$$
\left(2 p_{1}+2 k\right)_{n}=\frac{\Gamma\left(2 p_{1}+2 k+n\right)}{\Gamma\left(2 p_{1}+2 k\right)}
$$


Next, based on the properties of the gamma function we have the following

$$
\Gamma\left(2 p_{1}+2 k\right)=\pi^{-\frac{1}{2}} 2^{2 p_{1}+2 k-1} \Gamma\left(p_{1}+k\right) \Gamma\left(p_{1}+k+\frac{1}{2}\right)
$$

Which is equivalent with

$$
\Gamma\left(2 p_{1}+2 k\right)=\pi^{-\frac{1}{2}} 2^{2 p} 4^{k} \Gamma\left(p_{1}+k\right) \Gamma\left(p_{2}+k\right)
$$

Similarly, we need to simplify $\Gamma\left(2 p_{1}+2 k+n\right)$ as follows, for $n$ even and odd. First, for $n=2 m$ even we have the same result as the above for $k \rightarrow k+m$

$$
\Gamma\left(2 p_{1}+2 k+2 m\right)=\frac{2^{2 p_{4}^{k+m}} \Gamma\left(p_{1}+k+m\right) \Gamma\left(p_{2}+k+m\right)}{\sqrt{\pi}}
$$

and for $n=2 m+1$ odd we have

$$
\begin{aligned}
& \qquad\left(2 p_{2}+2 k+2 m\right)=\frac{2^{2 p_{1} 4^{k+m}} \Gamma\left(p_{2}+k+m\right) \Gamma\left(p_{3}+k+m\right)}{\sqrt{\pi}} \\
& \qquad \gamma\left(2 p_{1}+2 k, d x\right)=\frac{(d x)^{2 p_{1}+2 k}}{2\left(p_{1}+k\right)}\left[\sum_{n=2 m}^{\infty} \frac{\left(2 p_{1}+2 k\right)}{\left(2 p_{2}+2 k\right)}\right. \\
& \text { Next, substituting (37) and (7) into the first summation of (40) } \\
& \text { we obtain } \\
& \qquad \sum_{\substack{n=0 \\
n=2 m}}^{\infty} \frac{\left(2 p_{1}+2 k\right)_{2 m}(-d x)^{2 m}}{\left(2 p_{2}+2 k\right)_{2 m}(2 m) !}=\sum_{\substack{n=0 \\
n=2 m}}^{\infty} \frac{\left(p_{1}+k\right)_{m}(d x)^{2 m}}{\left(p_{3}+k\right)_{m}(2 m) !}
\end{aligned}
$$

$$
\gamma\left(2 p_{1}+2 k, d x\right)=\frac{(d x)^{2 p_{1}+2 k}}{2\left(p_{1}+k\right)}\left[\sum_{\substack{n=0 \\ n=2 m}}^{\infty} \frac{\left(2 p_{1}+2 k\right)_{2 m}(-d x)^{2 m}}{\left(2 p_{2}+2 k\right)_{2 m}(2 m) !}+\sum_{\substack{n=2 \\ n=2 m+1}}^{\infty} \frac{\left(2 p_{1}+2 k\right)_{2 m+1}(-d x)^{2 m+1}}{\left(2 p_{2}+2 k\right)_{2 m+1}(2 m+1) !}\right]
$$
we obtain

Which is identical with

$$
\sum_{n=2 m}^{\infty} \frac{\left(2 p_{1}+2 k\right)_{2 m}(-d x)^{2 m}}{\left(2 p_{2}+2 k\right)_{2 m}(2 m) !}=\sum_{m=0}^{\infty} \frac{\left(p_{1}+k\right)_{m}\left(d^{2} x^{2}\right)^{m}}{\left(p_{3}+k\right)_{m}\left(\frac{1}{2}\right)_{m} 4^{m} m !}
$$

of (40) yields

$$
\sum_{n=2 m+1}^{\infty} \frac{\left(2 p_{1}+2 k\right)_{2 m+1}(-d x)^{2 m+1}}{\left(2 p_{2}+2 k\right)_{2 m+1}(2 m+1) !}=\frac{-d x \sum_{m=0}^{\infty} \frac{\left(p_{2}+k\right) m(-d x)^{2 m}}{\left.\left(p_{4}+k\right)_{m}\left(\frac{3}{2}\right)_{m}\right)^{m_{(m) !}}}}{\left(p_{1}+k\right)^{-1}\left(p_{2}+k\right)}
$$

Which is equivalent with

$$
\sum_{n=2 m+1}^{\infty} \frac{\left(2 p_{1}+2 k\right)_{2 m+1}(-d x)^{2 m+1}}{\left(2 p_{2}+2 k\right)_{n}(2 m+1) !}=\frac{-d x\left(p_{1}+k\right) \sum_{m=0}^{\infty} \frac{\left(p_{2}+k\right) m\left(\frac{d^{2} x^{2}}{2^{2}}\right)^{m}}{\left(p_{4}+k\right) m\left(\frac{3}{2}\right)_{m} m !}}{\left(p_{2}+k\right)}
$$

Next, substituting (42) and (44) into (40) produces

Similarly, substituting (39) and (13) into the second summation

$$
\gamma\left(2 p_{1}+2 k, d x\right)=\frac{(d x)^{2 p_{1}+2 k}}{2}\left[\frac{1}{\left(p_{1}+k\right)} \sum_{m=0}^{\infty} \frac{\left(p_{1}+k\right)_{m}\left(\frac{d^{2} x^{2}}{2^{2}}\right)^{m}}{\left(p_{3}+k\right)_{m}\left(\frac{1}{2}\right)_{m} m !}-\frac{d x}{\left(p_{2}+k\right)} \sum_{m=0}^{\infty} \frac{\left(p_{2}+k\right)_{m}\left(\frac{d^{2} x^{2}}{2^{2}}\right)^{m}}{\left(p_{4}+k\right)_{m}\left(\frac{3}{2}\right)_{m} m !}\right]
$$

Substituting (45) into (88) of Progri 2016 [1] we obtain for $\alpha=2 p+2 k$

$$
F_{\mathrm{GBessel} 1}(x ; a, d, p)=\frac{\left(1-\frac{1}{d^{2}}\right)^{p_{1}}(d x)^{2 p_{1}}}{\Gamma\left(2 p_{1}\right)} \sum_{k=0}^{\infty} \frac{x^{2 k}\left[\frac{1}{\left(p_{1}+k\right)} \sum_{m=0}^{\infty} \frac{\left(p_{1}+k\right)_{m}\left(\frac{d^{2} x^{2}}{2^{2}}\right)^{m}}{\left(p_{3}+k\right)_{m}\left(\frac{1}{2}\right)_{m} m !}-\frac{d x}{\left(p_{2}+k\right)} \sum_{m=0}^{\infty} \frac{\left(p_{2}+k\right)_{m}\left(\frac{d^{2} x^{2}}{2^{2}}\right)^{m}}{\left(p_{4}+k\right)_{m}\left(\frac{3}{2}\right)_{m} m !}\right]}{2 \times k !\left(p_{2}\right)_{k} 2^{2 k}}
$$

Which is equivalent with the following

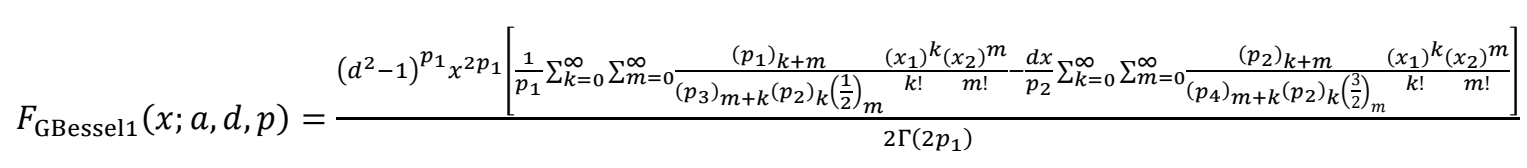

Where

$$
x_{1}=\frac{x^{2}}{4} ; x_{2}=\frac{x^{2} d^{2}}{4}
$$

The cdf of the Bessel function distribution of the first kind which can be written with the help of the Kampé de Fériet function (or double hypergeometric series) [8]-[10] as follows

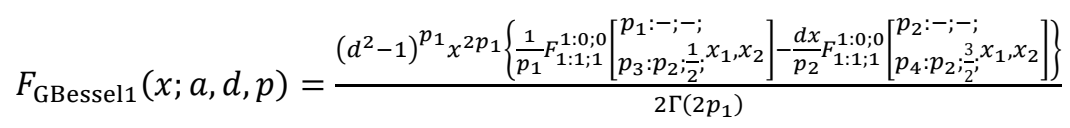


Which is equivalent with

$$
\begin{aligned}
F_{\mathrm{GBessel} 1}(x ; a, d, p)= & C_{11}(d, p) \mathcal{F}_{\mathrm{GBessel} 1}(x ; a, d, p)(50) \quad \text { given by } \\
& \mathcal{F}_{\mathrm{GBessel} 1}(x ; a, d, p)=\frac{x^{2 p_{1}} p_{1}\left\{\frac{1}{p_{1}} F_{1: 1 ; 1}^{1: 0 ; 0}\left[\begin{array}{l}
p_{1}:-;-; \\
p_{3}: p_{2} ; \frac{1}{2} ;
\end{array} x_{1}, x_{2}\right]-\frac{d x}{p_{2}} F_{1: 1 ; 1}^{1: 0 ; 0}\left[\begin{array}{l}
p_{2}:-;-; \\
p_{4}: p_{2} ; \frac{3}{2} ;
\end{array} x_{1}, x_{2}\right]\right\}}{\Gamma\left(p_{2}\right)}
\end{aligned}
$$

Before we investigate the efficient computation of the Generalized Bessel function distribution CDF of the second kind we must first answer the following question. Are both approaches of the numerical implementation of the computation of the Generalized Bessel function distribution CDF of the first kind equally efficient? Because one approach uses the sum of two Kampé de Fériet functions (or double hypergeometric series) [8]-[10] and the other one the difference.

The answer to this important question is already given in the endnotes.

\section{Numerical, Theoretical Results}

Progri 2016 [1] numerical, theoretical results section contains the first set of simulation results of pdfs and approximated numerical cdfs of the generalized Bessel function distributions of the first and second kinds based analytical derivations presented in Progri 2016 [1]; i.e., in Progri 2016, we did not provide any simulation results of the exact closed form expression of the cdfs of the generalized Bessel function distributions of the first and second kinds.

Hence, in this section we present for the first time the MATLAB simulation results of the of the exact closed form expression of the cdfs of the generalized Bessel function distributions of the first and second kinds based on closed form expressions (117) and (142) in Progri 2016 and (25) in the current journal paper.

Moreover, we want to ensure the reader that when we produced the simulation results of this section every single equation of Progri 2016 and of the current journal paper has been tested; hence, the simulation results go far beyond the simulation results of just closed form expressions (117) and (142) in Progri 2016 and (25) in the current journal paper.

\subsection{Generalized Bessel Function Distribution of the First and Second Kinds}

In this subsection we present, numerical results of the generalized Bessel function of the first and second kinds using the exact closed form expression found in this paper and in Progri 2016 [1].
Where $C_{11}(d, p)$ is given by $(26)$ and $\mathcal{F}_{\mathrm{GBessel}}(x ; a, d, p)$ is

Figure 1(a) shows the plots of (top) the generalized Bessel of the first kind pdf and cdf for $a=1, d=1.6$, and $p=3.1$ for the integral and linear numerical approximation; (bottom) cdf using (25) in the current journal paper and (116) or $(117)^{\mathrm{v}}$ from Progri 2016 [1].

Figure 1(b) shows exactly the same results as the ones in Fig. 1(a) but for the generalized Bessel of the second kind pdf and cdf for $a=1, d=1.6$, and $p=3.1$.

The important results here are that even for non-integer values of $p$ the generalized Bessel of the first and second kinds pdfs and cdfs are validated.

\subsection{Absolute Errors of the Generalized Bessel Function Distribution of the First and Second Kinds}

Absolute errors of the generalized Bessel function distribution of the first and second kinds are shown in Fig. 2.

Figure 2 (a) shows absolute errors of the generalized Bessel of the first kind cdf for $a=1, d=1.6, p=3.1$. Figure 2 (b) shows absolute errors of the generalized Bessel of the second kind cdf for $a=1, d=1.6, p=3.1$; i.e., $p$ not an integer.

\section{Conclusions}

The main focus on this journal paper is to validate the entire derivations of the closed form expressions provided in Progri 2016 [1] namely: (1) the cdf of the generalized Bessel of the first kind cdf, (116) or (117) from Progri 2016 [1] and (25) in the current journal paper; (2) the cdf of the generalized Bessel of the second kind cdf for non-integer values of $p,(142)$ from Progri 2016 [1].

We can say with absolute certainty that all the closed form expressions provided in Progri 2016 [1] have been simulated and tested. The numerical results obtained from the closed form expression in Fig. 2 are several orders of magnitude more accurate than those obtained from the linear approximation simulation results presented initially in Progri 2016 [1].

Numerical results are derived for each case to validate the theoretical models presented in the paper. 


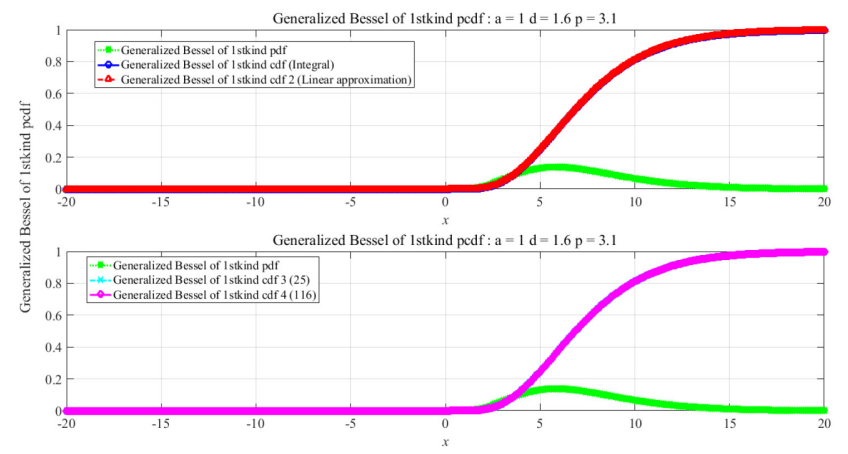

(a) (top) original generalized Bessel of the first kind pdf and cdf for $a=1, d=1.6$, and $p=3.1$, for the integral and linear numerical approximation; (bottom) original generalized Bessel of the first kind pdf and cdf for $a=1, d=1.6$, and $p=3.1$ for (25) and (116)/(117) from Progri 2016 [1].

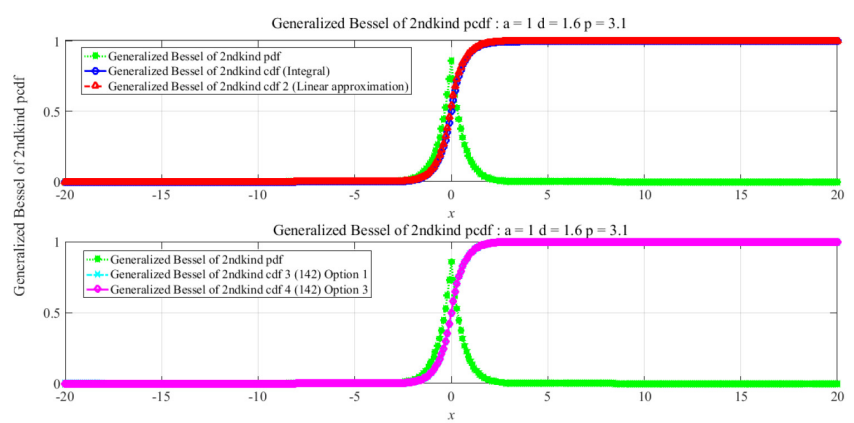

(b) (top) original generalized Bessel of the second kind pdf and cdf for $a=1, d=1.6$, and $p=3.1$ for the integral and linear numerical approximation; (bottom) original generalized Bessel of the second kind pdf and cdf for $a=1, d=1.6$, and $p=3.5$, for (142) option 1 and (142) option 3 of Kampé de Fériet function from Progri 2016 [1]; $p$ not an integer.

FIGURE 1: Numerical calculations of the generalized Bessel of the (a) first and (b) second kind pdf and cdf vs. Generalized Bessel of the (a) first and (b) second kind: original pdf and cdf: $p$ not an integer based on the computation of Kampé de Fériet function.

For each generalized Bessel distribution model the closed form expression of cdf is given by means of series expansion of the modified Bessel functions which leads to incomplete gamma functions, hypergeometric series, and the Kampé de Fériet function.

It is also shown that for most practical applications the generalized Bessel function distribution cdf of the first and second kinds can be represented in closed form by means of the family of well-known the Kampé de Fériet functions to within six to fifteen orders of magnitude accuracy.

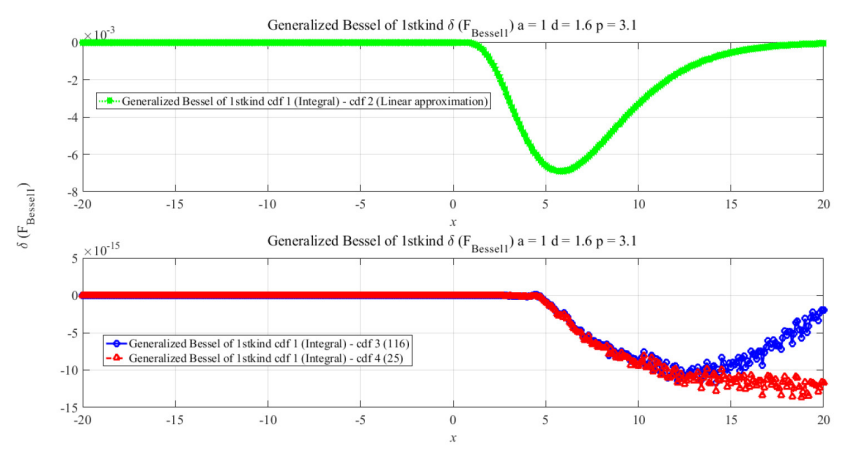

(a) (top) absolute error of the original generalized Bessel of the first kind pdf and cdf for $a=1, d=1.6$, and $p=3.1$ integral and linear numerical approximation; (bottom) the original generalized Bessel of the first kind pdf and closed form expression of the cdf, for for $a=1, d=1.6$, and $p=3.1$ (25) and (116)/(117) from Progri 2016 [1].

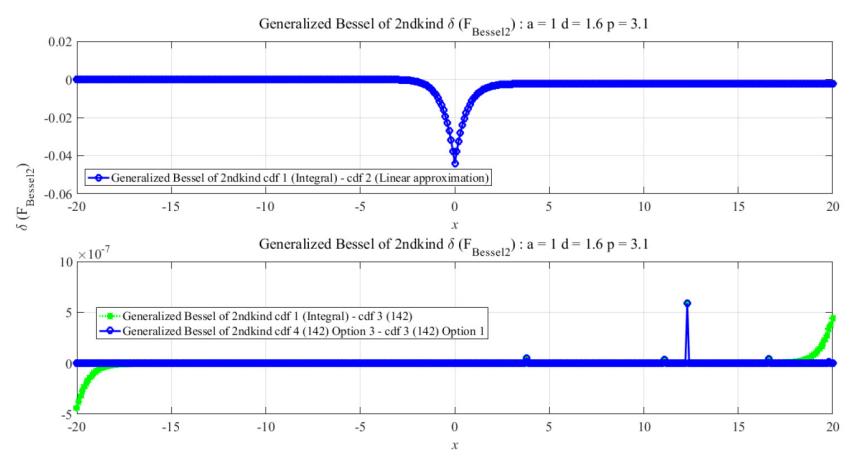

(b) (top) absolute error of the original generalized Bessel of the second kind pdf and cdf for $a=1, d=1.6$, and $p=3.1$, for the integral and linear numerical approximation; (bottom) absolute error of the original generalized Bessel of the second kind pdf and cdf for $a=1, d=1.6$, and $p=3.5$, for (142) option 1 and (142) option 3 of Kampé de Fériet function from Progri 2016 [1]; $p$ not an integer.

FIGURE 2: Numerical calculations of the absolute error of the generalized Bessel of the first and second kind pdf and cdf vs. approximated Bessel of the first and second kind pdf and cdf.

\section{Acknowledgement}

This work was supported by Giftet Inc. executive office.

\section{References}

[1] I. Progri, "Generalized Bessel function distributions," $J$. Geol. Geoinfo. Geointel., vol. 2016, article ID 2016071602, 15 pg., Nov. 2016. DOI: 
http://doi.org/10.18610/JG3.2016.071602,

http://giftet.com/JG3/2016/071602.pdf.

[2] I.F. Progri, P. Huang, Y. Pi, X. Xia, "Indoor adaptive GNSS signal acquisition. Part 1: theory and simulations," J. Geol. Geoinfo. Geointel., vol. 2016, article ID 2016071601, 19 pg., Nov. 2016. DOI: http://doi.org/10.18610/JG3.2016.071601, http://giftet.com/JG3/2016/071601.pdf.

[3] 1. Progri, I., Huang, P., Pi, Y., Xia, X., "Indoor adaptive GNSS signal acquisition - theory and simulations," $J$. Geol. Geoinfo. Geointel., vol. 2016, article ID 2016071601, 19 pg., Nov. 2016. DOI: http://doi.org/10.18610/JG3.2016.071601, http://giftet.com/JG3/2016/071601.pdf.

[4] I. Progri, "Advanced anti-jam indoor adaptive GNSS signal acquisition: part 1, normal distribution-theory and simulations," J. Geol. Geoinfo. Geointel., vol. 2018, article ID 2018071604, 26 pg., Nov. 2018. DOI: http://doi.org/10.18610/JG3.2018.071604, http://giftet.com/JG3/2018/071604.pdf.

[5] I. Progri, "Maximum likelihood GNSS parameter estimation: part 2, Bessel distribution:-theory and simulation," in Proc. ION-ITM, Reston, VA, pp. 589-610, Jan. 29-Feb. 1, 2018.

[6] I. Progri, "Advanced anti-jam indoor adaptive GNSS signal acquisition: part 2, Bessel distribution-Theory," in Proc. ION-ITM, Reston, VA, pp. 440-466, Jan. 29-Feb. 1, 2018.

[7] Anon, "Kampé de Fériet function," Wikipedia, the free encyclopedia, Dec. 2015, https://en.wikipedia.org/wiki/Kamp\%C3\%A9_de_F\%C3 $\%$ A9riet_function.

[8] P. Appell, J., Kampé de Fériet, Fonctions Hypergéométriques et Hypersphériques; Polynomes d'Hermites. Paris: Gauthier-Villars, 1926.

[9] S. Ali, "On a reducibility of the Kampé de Fériet function," Adv. Info. Techn. Manag., vol. 1, no. 1, pp. 24-27, Mar. 2012.

[10] S. Ali, "A transformation formula for the Kampé de Fériet function" Inter. Conf. Ceramics, Intern. J. Modern Physics: Conf. Series, vol. 22, pp. 713-719, 2013, DOI: http://doi.org/10.1142/S2010194513010908.

[11]P. Agarwal, M. Chand, "Graphical analysis of Kampé De Fériet's series with implementation of MATLAB," Intern. J. Comp. Applic. (0975-8887), vol. 59, no. 4, Dec. 2012.
[12] Anon, "Incomplete Gamma function," Wikipedia, the free encyclopedia, Dec.

2015 , http://en.wikipedia.org/wiki/Incomplete_gamma_function.

[13]H. Bateman, Higher Transcendental Functions, vols. I, II, and III, (California Institute of Technology Bateman Manuscript Project, Dir. Arthur Erdélyi et al.) New York: McGraw-Hill, 1953-1955.

[14] Anon., "Taylor series," Wikipedia, the free encyclopedia, Dec. 2016, https://en.wikipedia.org/wiki/Taylor_series.

[15] Anon., "Brook Taylor," Wikipedia, the free encyclopedia, Dec. 2016, https://en.wikipedia.org/wiki/Brook_Taylor.

[16] A.M. Legendre, Essai sur la théorie des nombres, Dupart, Paris, An VI, 1798.

[17] A.M. Legendre, Exercises de calcul integral, Paris, 1811.

[18] Anon., "Adrien-Marie Legendre," Wikipedia, the free encyclopedia,

Dec.

2016 , https://en.wikipedia.org/wiki/Adrien-Marie_Legendre.

[19]D. Barbolosi, H. Jager, "On a theorem of Legendre in the theory of continued fractions," $J$. de théorie des nombres de Bordeaux, vol. 6, no. 1, pp. 81-94, 1994, URL: http://numdam.org/article/JTNB_1994_6_1_81_0.pdf.

[20] K. Pearson, "Tables of the incomplete gamma-function," Nature, vol. 110, pp 669-, Nov. 1922, DOI: https://doi.org/10.1038/110669c0.

[21] W. Gautschi "A computational procedure for incomplete gamma functions," ACM Trans. Math. Software, vol. 5, no. 4, pp. 482- 489, Dec. 1979.

[22] N.M. Temme, "The asymptotic expansion of the incomplete gamma functions," SIAM J. Math. Analy. vol. 10, no. 4, pp. 757-766, 1979, DOI: https://doi.org/10.1137/0510071.

[23] W.B. Jones, W.J. Thron, "On the computation of incomplete gamma functions in the complex domain," $J$. Comp. \& Appl. Math., vol. 12 \& 13, pp. 401-417, 1985, DOI: https://doi.org/10.1016/0377-0427(85)90034-2.

[24] N.M. Temme, "On the computation of the incomplete gamma functions for large values of the parameters," Private Commun., 11 pg., 1985, researchgate.net.

[25] A.R., DiDonato, A.H. Morris Jr., "Computation of the incomplete gamma function ratios and their inverse," $A C M$ Trans. Math. Soft. (TOMS), vol. 12, no. 4, pp. 377-393, 1986.

[26] N.M. Temme "Uniform asymptotics for the incomplete gamma functions starting from negative values of the 
parameters," Methods \& Appl. Analy. vol. 3, no. 3, pp. 335344, 1996.

[27] S. Winitzki "Computing the incomplete Gamma function to arbitrary precision." in: V. Kumar, M.L. Gavrilova, C.J.K. Tan, P. L'Ecuyer (eds) Comp. Science \& Appl. ICCSA 2003. ICCSA 2003. Lecture Notes in Computer Science, vol. 2667, pp. 790-798. Springer, Berlin, Heidelberg, 2003.

[28] U. Blahak, "Efficient approximation of the incomplete gamma function for use in cloud model applications," Geoscien. Mod. Devel., vol. 3, no. 2, pp. 329-336, Jul. 2010, DOI: https://doi.org/10.5194/gmd-3-329-2010.

[29]I. Thompson, “Algorithm 926: Incomplete Gamma functions with negative arguments," ACM Trans. Math. Soft., vol. 39, no. 2, Feb. 2013, DOI: http://doi.org/10.1145/2427023.2427031.

[30] D.H. Bailey, J.M. Borwein, “Crandall's computation of the incomplete Gamma function and the Hurwitz zeta function, with applications to Dirichlet L-series," Applied Math. \& Comp., vol. 268, pp 462-477, Jan. 2015, DOI: https://doi.org/10.1016/j.amc.2015.06.048.

[31] R. Abergel, L. Moisan, "Fast and accurate evaluation of a generalized incomplete gamma function," MAP5 2016-14, pp 1-29, 2016. URL: https://hal.archives-ouvertes.fr/hal01329669.

[32] G.J.O. Jameson, "The incomplete gamma functions," The Mathematical Gazette, vol. 100, no. 548, pp. 298-306, Jul. 2016, DOI: https://doi.org/10.1017/mag.2016.67.

[33]A. Gil, D. Ruiz-Antolín, J. Segura, N.M. Temme, "Algorithm 969: computation of the incomplete gamma function for negative values of the argument," ACM Trans. Math. Soft. (TOMS), vol. 43, no. 3, pp. 1-28, Jan. 2017, DOI: https://doi.org/10.1145/2972951.

[34]P. Greengard, V. Rokhlin, "An algorithm for the evaluation of the incomplete gamma function," $A d v$ Comput. Math., pp 1-27, Mar. 2018. https://doi.org/10.1007/s10444-018-9604-х.

[35] I. Progri, Indoor Geolocation Systems-Theory and Applications. Vol. I, $1^{\text {st }}$ ed., Worcester, MA: Giftet Inc., $\sim 800$ pp., $\sim 2018$ (not yet available in print).

[36] I.S. Gradshteyn, I.M. Ryzhik, (A. Jeffrey, D. Zwillinger, editors) Table of Integrals, Series, and Products, 7th ed., Burlington, MA: Academic Press, 1171 pp., 2007.

$$
F_{g: c ; d}^{h: a ; b}\left[\begin{array}{l}
(H)_{h}:(A)_{a} ;(B)_{b} ; x, y \\
(G)_{g}:(C)_{c} ;(D)_{d} ;
\end{array} ;=\sum_{m, n \geq 0}^{\infty} \frac{\left(\left(H_{h}\right)\right)_{m+n}\left(\left(A_{a}\right)\right)_{m}\left(\left(B_{b}\right)\right)_{n}}{\left(\left(G_{h}\right)\right)_{m+n}\left(\left(C_{c}\right)\right)_{m}\left(\left(D_{d}\right)\right)_{n}} \frac{x^{m}}{m !} \frac{y^{m}}{n !}\right.
$$

[37]Anon, "Gamma function," Wikipedia, the free encyclopedia, Dec. 2015, https://en.wikipedia.org/wiki/Gamma_function.

[38] Anon, "Hypergeometric function," Wikipedia, the free encyclopedia, 2015, https://en.wikipedia.org/wiki/Hypergeometric_function.

[39]Anon, "Pochhammer symbol," Wikipedia, the free encyclopedia, 2015, https://en.wikipedia.org/wiki/Pochhammer_symbol.

[40]Anon, "MATLAB 2016b," The MathWorks, Inc., Natick, MA, Copyright (C) 1994-2016, The MathWorks, Inc., http://mathworks.com/products/new_products/release200 6b.html?requestedDomain=mathworks.com.

[41]L.R. Arnaut, "Limit distributions for imperfect electromagnetic reverberation," IEEE Trans. Electr. Comp., vol. 45, no. 2, pp. 357-377, May 2003. DOI: http://doi.org/10.1109/TEMC.2003.811301.

[42]Anon, "Generalized Hypergeometric function," Wikipedia, the free encyclopedia, June, 2017, https://en.wikipedia.org/wiki/Generalized_hypergeometri c_function.

\section{Appendix A: A Super-Efficient Implementation of the Kampé de Fériet Function or Double Hypergeometric Series}

The main purpose of this section is to develop a supper efficient implementation algorithm of the Kampé de Fériet double hypergeometric series is defined as [1]-[10] that accomplishes the following three criteria: it is numerically very stable, computationally very efficient, and numerically very accurate; i.e., the Kampé de Fériet function numerical evaluation in MATLAB is no longer impacted by the numerical stability, efficiency, and accuracy. I hope that the Kampé de Fériet function, presented in this appendix, can be implemented in MATLAB in like manner as the generalized hypergeometric series function hypergeom(a,b,z) [40].

The Kampé de Fériet double hypergeometric series is defined as [1]-[10] 
A super-efficient implementation of the Kampé de Fériet function or double hypergeometric series [1]-[10] should be able to take advantage of the super-fast implementation of the generalized hypergeometric function [38], [42]; i.e., reduce the computation of the Kampé de Fériet double hypergeometric series [1]-[10] into the computation of a single summation.

For that we need to write the definition of the generalized hypergeometric series as follows [42]

$$
{ }_{a} F_{c}\left[\begin{array}{l}
(A)_{a} \\
(C)_{c}
\end{array}\right]={ }_{a} F_{c}\left[\begin{array}{l}
A_{1}, \cdots, A_{a} \\
C_{1}, \cdots, C_{c} x
\end{array}\right]={ }_{a} F_{c}\left[A_{1}, \cdots, A_{a} ; C_{1}, \cdots, C_{c} ; x\right]=\sum_{m \geq 0}^{\infty} \frac{\left(\left(A_{a}\right)\right)_{m}}{\left(\left(C_{c}\right)\right)_{m}} \frac{x^{m}}{m !}=\sum_{m \geq 0}^{\infty} \frac{\left(A_{1}\right)_{m} \cdots\left(A_{a}\right)_{m}}{\left(C_{1}\right)_{m} \cdots\left(C_{c}\right)_{m}} \frac{x^{m}}{m !}
$$

Employing a well-known identity of the Pochhammer symbol [39] into the formulation of the Kampé de Fériet double hypergeometric series [1]-[10] can be written as

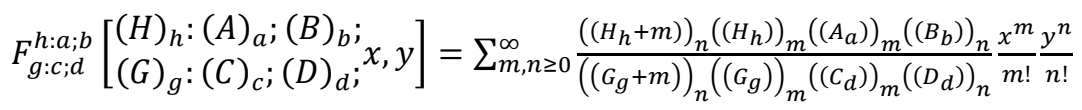

Equation (54) can be split into two summations

$$
F_{g: c ; d}^{h: a ; b}\left[\begin{array}{l}
(H)_{h}:(A)_{a} ;(B)_{b} ; \\
(G)_{g}:(C)_{c} ;(D)_{d} ;
\end{array} ;, y\right]=\sum_{m \geq 0}^{\infty} \frac{\left(\left(H_{h}\right)\right)_{m}\left(\left(A_{a}\right)\right)_{m}}{\left(\left(G_{g}\right)\right)_{m}\left(\left(C_{c}\right)\right)_{m}}\left[\sum_{n \geq 0}^{\infty} \frac{\left(\left(H_{h}+m\right)\right)_{n}\left(\left(B_{a}\right)\right)_{n}}{\left(\left(G_{g}+m\right)\right)_{n}\left(\left(D_{d}\right)\right)_{n}} \frac{y^{n}}{n !}\right] \frac{x^{m}}{m !}
$$

The inner hypergeometric series can be written as

${ }_{h, b} F_{g, d}\left[\begin{array}{l}(H+m)_{h}(B)_{b} y \\ (G+m)_{g}(D)_{d} y\end{array}\right]=\sum_{n \geq 0}^{\infty} \frac{\left(\left((H+m)_{h}\right)\right)_{n}\left(\left(B_{b}\right)\right)_{n}}{\left(\left((G+m)_{g}\right)\right)_{n}\left(\left(D_{d}\right)\right)_{n}} \frac{y^{m}}{n !}(56)$

Substituting (56) into (55) yields a 1-dimensional supper fast and accurate summation of the Kampé de Fériet double hypergeometric series [1]-[10] as follows

$$
F_{g: c ; d}^{h: a ; b}\left[\begin{array}{l}
(H)_{h}:(A)_{a} ;(B)_{b} ; x, y \\
(G)_{g}:(C)_{c} ;(D)_{d} ;
\end{array} x=\sum_{m \geq 0}^{\infty} \frac{\left(\left(H_{h}\right)\right)_{m}\left(\left(A_{a}\right)\right)_{m}}{\left(\left(G_{g}\right)\right)_{m}\left(\left(C_{c}\right)\right)_{m}}{ }_{h, b} F_{g, d}\left[\begin{array}{l}
(H+m)_{h}(B)_{b} \\
(G+m)_{g}(D)_{d}
\end{array} y\right] \frac{x^{m}}{m !}\right.
$$

Next, we need a computationally efficient algorithm of $\left(\left(H_{h}\right)\right)_{m},\left(\left(A_{a}\right)\right)_{m},\left(\left(G_{g}\right)\right)_{m}$, and $\left(\left(C_{c}\right)\right)_{m}$.

Let us consider for example the computation of $\left(\left(A_{a}\right)\right)_{m}$

$$
\left(\left(A_{a}\right)\right)_{m}=\prod_{i=1}^{a}\left(A_{i}\right)_{m}=\prod_{i=1}^{a} \frac{\Gamma\left(A_{i}+m\right)}{\Gamma\left(A_{i}\right)}
$$

For large values of $m \gg 1$ and $A_{i} \gg 1, \Gamma\left(A_{i}+m\right) \rightarrow \infty$; hence, an efficient computation of $\left(\left(A_{a}\right)\right)_{m} /\left(\left(C_{c}\right)\right)_{m}$ is required.

From the definition of the Pochhammer symbols $\left(A_{i}\right)_{m}$ [39] we have the following

$$
\left(A_{i}\right)_{m}=\prod_{k=0}^{m-1}\left(A_{i}+k\right)
$$

Substituting (59) into (58) yields

$$
\left(\left(A_{a}\right)\right)_{m}=\prod_{i=1}^{a} \prod_{k=0}^{m-1}\left(A_{i}+k\right)=\prod_{k=0}^{m-1} \prod_{i=1}^{a}\left(A_{i}+k\right)(60)
$$

Similarly we can write exactly the same formulas for $\left(\left(H_{h}\right)\right)_{m},\left(\left(G_{g}\right)\right)_{m}$, and $\left(\left(C_{c}\right)\right)_{m}$ as follows

$$
\begin{aligned}
& \left(\left(H_{h}\right)\right)_{m}=\prod_{i=1}^{h} \prod_{k=0}^{m-1}\left(H_{i}+k\right)=\prod_{k=0}^{m-1} \prod_{i=1}^{h}\left(H_{i}+k\right) \\
& \left(\left(G_{g}\right)\right)_{m}=\prod_{i=1}^{g} \prod_{k=0}^{m-1}\left(G_{i}+k\right)=\prod_{k=0}^{m-1} \prod_{i=1}^{g}\left(G_{i}+k\right)
\end{aligned}
$$

$$
\left(\left(C_{c}\right)\right)_{m}=\prod_{i=1}^{c} \prod_{k=0}^{m-1}\left(C_{i}+k\right)=\prod_{k=0}^{m-1} \prod_{i=1}^{c}\left(C_{i}+k\right)
$$

Before we can perform the computation of (57) can we write the following term as:

$$
\frac{\left(\left(H_{h}\right)\right)_{m}\left(\left(A_{a}\right)\right)_{m}}{\left(\left(G_{g}\right)\right)_{m}\left(\left(C_{c}\right)\right)_{m}}=\frac{\prod_{k=0}^{m-1} \prod_{i=1}^{h}\left(H_{i}+k\right) \prod_{k=0}^{m-1} \prod_{i=1}^{a}\left(A_{i}+k\right)}{\prod_{k=0}^{m-1} \prod_{i=1}^{g}\left(G_{i}+k\right) \prod_{k=0}^{m-1} \prod_{i=1}^{c}\left(C_{i}+k\right)}
$$

Which can be further simplified as follows:

$$
\frac{\left(\left(H_{h}\right)\right)_{m}\left(\left(A_{a}\right)\right)_{m}}{\left(\left(G_{g}\right)\right)_{m}\left(\left(C_{c}\right)\right)_{m}}=\prod_{k=0}^{m-1} \frac{\prod_{i=1}^{h}\left(H_{i}+k\right)}{\prod_{i=1}^{g}\left(G_{i}+k\right)} \frac{\prod_{i=1}^{a}\left(A_{i}+k\right)}{\prod_{i=1}^{c}\left(C_{i}+k\right)}
$$

If we define $q_{k}$ as below

$$
q_{k}=\frac{\prod_{i=1}^{h}\left(H_{i}+k\right)}{\prod_{i=1}^{g}\left(G_{i}+k\right)} \frac{\prod_{i=1}^{a}\left(A_{i}+k\right)}{\prod_{i=1}^{c}\left(C_{i}+k\right)}
$$

Then we can eliminate the singularity in the computation of the Kampé de Fériet double hypergeometric series [1]-[10] as follows

$$
\frac{\left(\left(H_{h}\right)\right)_{m}\left(\left(A_{a}\right)\right)_{m}}{\left(\left(G_{g}\right)\right)_{m}\left(\left(C_{c}\right)\right)_{m}}=\prod_{k=0}^{m-1} q_{k}
$$

Next, by defining

$$
Q_{m}=\prod_{k=0}^{m-1} q_{k} ; \tilde{Q}_{m}(x)=\prod_{k=1}^{m} \frac{q_{k-1} x}{k}
$$


By substituting (68) into (66), then (65) and then (57) yields

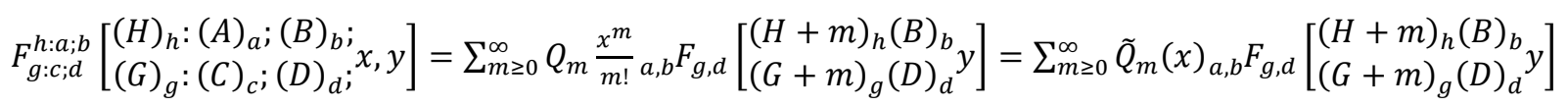

Finally, let us try to re-compute (54) or (55) using the technique that we just demonstrated in (68).

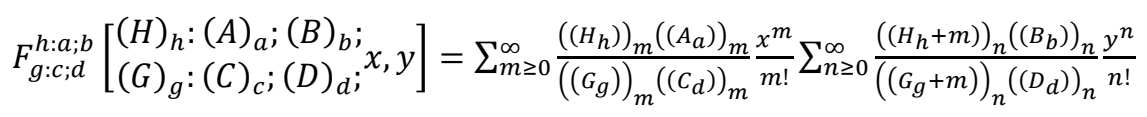

This concludes the super-efficient computation of the Kampé de Fériet function or double hypergeometric series [1]-[10]. It is numerically very stable, computationally very efficient, and extremely accurate. I hope that an efficient implementation of

i Dr. Progri first begun to work on the cdf of the generalized Bessel function distributions as early as 2015 [2] and all the way to 2018 [6] and has reported his work in various publications. A couple of the early publications [3], [4] do not contain the fully tested closed form cdfs.

ii In mathematics, a Taylor series is a representation of a function as an infinite sum of terms that are calculated from the values of the function's derivatives at a single point [14]. The concept of a Taylor series was formulated by the Scottish mathematician James Gregory and formally introduced by the English mathematician Brook Taylor [15] in 1715. If the Taylor series is centered at zero, then that series is also called a Maclaurin series, named after the Scottish mathematician Colin Maclaurin, who made extensive use of this the Kampé de Fériet function in MATLAB can be performed in like manner as the generalized hypergeometric series function hypergeom $(\mathrm{a}, \mathrm{b}, \mathrm{z})[40]$.

special case of Taylor series in the 18th century.

iii I can say for sure that the expansion based on Legendre continued fraction is the most computationally efficient expansion in the literature [13]-[34].

iv The Taylor series expansion is less efficient; i.e., it requires a lot of terms to converge, and not event accurate; i.e., the results can be astronomically wrong for large values of $x$. There are a number of references [13]-[34] that support this argument.

v In all three scenarios generalized Bessel of the first kind pdf and cdf, (25) in the current journal paper and (116) or (117) from Progri 2016 [1], are in fact valid pdfs and cdfs. 